Mon. Not. R. Astron. Soc. 000,14(2016) Printed Monday $12^{\text {th }}$ November, $2018 \quad$ (MN LATEX style file v2.2)

\title{
Gauge Choice in Conformal Gravity
}

\author{
Joseph Sultana, ${ }^{1 \star}$ Demosthenes Kazanas, ${ }^{2} \dagger$ \\ ${ }^{1}$ Department of Mathematics, Faculty of Science, University of Malta, Msida MSD 2080, Malta \\ 2 Astrophysics Science Division, NASA/Goddard Space Flight Center, Greenbelt, Maryland 207r1, USA
}

\begin{abstract}
In a recent paper (MNRAS 458, $4122(2016))$ K. Horne examined the effect of a conformally coupled scalar field (referred to as Higgs field) on the Mannheim-Kazanas metric $g_{\mu \nu}$, i.e. the static spherically symmetric metric within the context of conformal gravity (CG), and studied its effect on the rotation curves of galaxies. He showed that for a Higgs field of the form $S(r)=S_{0} a /(r+a)$, where $a$ is a radial length scale, the equivalent Higgs-frame Mannheim-Kazanas metric $\tilde{g}_{\mu \nu}=\Omega^{2} g_{\mu \nu}$, with $\Omega=$ $S(r) / S_{0}$, lacks the linear $\gamma r$ term, which has been employed in the fitting of the galactic rotation curves without the need to invoke dark matter. In this brief note we point out that the representation of the Mannheim-Kazanas metric in a gauge where it lacks the linear term has already been presented by others, including Mannheim and Kazanas themselves, without the need to introduce a conformally coupled Higgs field. Furthermore, Horne argues that the absence of the linear term resolves the issue of light bending in the wrong direction, i.e. away from the gravitating mass, if $\gamma r>0$ in the Mannheim-Kazanas metric, a condition necessary to resolve the galactic dynamics in the absence of dark matter. In this case we also point out that the elimination of the linear term is not even required because the sign of the $\gamma r$ term in the metric can be easily reversed by a simple gauge transformation, and also that the effects of this term are indeed too small to be observed.
\end{abstract}

Key words: gravitation - galaxies:kinematics and dynamics - cosmology: theory, dark matter, dark energy

\section{Introduction}

Conformal (Weyl) gravity adopts the principle of local conformal invariance of spacetime under local conformal stretching $g_{\mu \nu}(x) \rightarrow \Omega^{2}(x) g_{\mu \nu}(x)$, where $\Omega(x)$ is a smooth strictly positive function. This is used as the supplementary condition that fixes the gravitational action, instead of requiring that the theory be no higher than second order as in the case of the Einstein-Hilbert action. This restrictive conformal invariance leads to a fourth order theory with the unique conformally invariant action

$$
\begin{aligned}
I_{W}= & -\frac{1}{2 \alpha} \int d^{4} x(-g)^{1 / 2} C_{\lambda \mu \nu \kappa} C^{\lambda \mu \nu \kappa} \\
= & -\frac{1}{\alpha} \int d^{4} x(-g)^{1 / 2}\left[R_{\mu \kappa} R^{\mu \kappa}-\left(R_{\nu}^{\nu}\right)^{2} / 3\right] \\
& + \text { a total derivative, }
\end{aligned}
$$

where $C_{\lambda \mu \nu \kappa}$ is the conformal Weyl tensor and $\alpha$ is a purely dimensionless coefficient. Varying the action in (2) with respect to the metric leads to the field equations

\footnotetext{
* E-mail: joseph.sultana@um.edu.mt

† E-mail: Demos.Kazanas-1@nasa.gov
}

$\frac{1}{\sqrt{-g}} g_{\mu \alpha} g_{\nu \beta} \frac{\delta I_{W}}{\delta g_{\alpha \beta}}=-\frac{1}{\alpha} W_{\mu \nu}$,

where

$W_{\mu \nu}=2 C_{\mu \nu ; \beta \alpha}^{\alpha \beta}+C_{\mu \nu}^{\alpha \beta} R_{\alpha \beta}$.

In the presence of sources the full field equations are obtained by variation with respect to the metric of the total action $I=I_{W}+I_{M}$, where $I_{M}$ is the action corresponding to the source. This gives

$W_{\mu \nu}=\frac{\alpha}{2} T_{\mu \nu}$,

where $T_{\mu \nu}=2(-g)^{-1 / 2} \delta I_{M} / \delta g^{\mu \nu}$ is a conformally invariant stress-energy tensor. It can be shown (Mannheim \& Kazanas 1989) that the tensor $W_{\mu \nu}$ vanishes when $R_{\mu \nu}$ is zero, so that any vacuum solution of Einstein's field equations is also a vacuum solution of Weyl gravity, with the converse not being necessarily true. Despite the highly nonlinear character of the field equations, a number of exact solutions (Mannheim \& Kazanas 1989, 1991; Mannheim 1990, 1992; Mannheim \& Kazanas 1994; Said, Sultana \& Zarb Adami 2012a b) of conformal Weyl gravity have been found. The Mannheim-Kazanas (MK) metric is the spherically symmetric vacuum solution in 
conformal gravity, satisfying $W_{\mu \nu}=0$ and describing the geometry outside a spherical body. This is given, up to a conformal factor, by the metric Mannheim \& Kazanas 1989)

$d s^{2}=-B(r) d t^{2}+\frac{d r^{2}}{B(r)}+r^{2}\left(d \theta^{2}+\sin ^{2} \theta d \phi^{2}\right)$,

where

$B(r)=1-\frac{\beta(2-3 \beta \gamma)}{r}-3 \beta \gamma+\gamma r-k r^{2}$,

and $\beta, \gamma$, and $k$ are integration constants. This solution includes the Schwarzschild $(\gamma=k=0)$ and the Schwarzschildde Sitter $(\gamma=0)$ solutions as special cases, with the latter requiring the presence of a cosmological constant in Einstein gravity. The constant $\gamma$, whose origin remains uncertain, has dimensions of acceleration; as such, conformal gravity is the unique (to our knowledge) gravitational theory that provides a solution with a characteristic, constant, Rindler-like acceleration, without the need to introduce one in the Lagrangian (such as the relativistic implementation of MOND, TeVeS (Bekenstein 2004)). Its magnitude is heuristically estimated to be $\mathrm{cH}_{0}$, on the basis of the asymptotically non-flat form of the resulting metric, a value consistent with the most recent observations of dynamics of galaxies (McGaugh 2016). An important consequence of this linear term in the metric which generated a growing interest in conformal gravity, has been the fitting of galactic rotational curves (Mannheim \& Kazanas 1989; Mannheim 1993, 1997; Mannheim \& O'Brien 2011, 2012) without the need to assume dark matter as in Einstein's gravitational theory. This is achieved (Mannheim \& Kazanas 1989) by associating $\gamma$ with the inverse Hubble length, i.e. $\gamma \simeq 1 / R_{H}$, such that the effects of this acceleration are comparable to those due to the Newtonian potential term $2 \beta / r \equiv r_{s} / r$ ( $r_{s}$ is the Schwarzschild radius), on length scales given by

$r_{s} / r^{2} \simeq \gamma \simeq 1 / R_{H}$ or $r \simeq\left(r_{s} R_{H}\right)^{1 / 2}$.

For example in the case of a galaxy of mass $M \simeq 10^{11} \mathrm{M}_{\odot}$ with $r_{s} \simeq 10^{16} \mathrm{~cm}$ and $R_{H} \simeq 10^{28} \mathrm{~cm}$, this scale is $r \sim 10^{22}$ $\mathrm{cm}$, i.e. roughly the size of the galaxy. Eq. (8) doesn't fix a particular length scale but represents a continuum of scales at which the contribution from the linear term becomes significant. Objects along this sequence do not include only galaxies but, at larger scales also galaxy clusters and at lower scales globular clusters, only recently found to require the presence of dark matter in order to account for the observed dynamics Scarpa, Marconi \& Gilmozzi 2003; Kazanas 2009). Besides the fitting of galactic rotational curves the effect of the linear $\gamma r$ term in the MKmetric (7) on the classical tests, such as the bending of light (Ederv \& Paraniape 1998; Amore, Arceo \& Fernandez 2006; Sultana \& Kazanas 2010) and perihelion precession (Sultana, Kazanas \& Said 2012) have been investigated.

In the next section we discuss the results of Horne (2016) and point out that the presence of a conformally coupled scalar field to the theory is not required to eliminate the linear term from the MK-metric. Moreover in section 3 we show that for light bending one can still achieve a positive deflection from the linear term by choosing an appropriate gauge. We summarize our results in the conclusion.

\section{The choice of gauge}

Brihaye \& Verbin (2009) obtained an exact static spherically symmetric solution to scalar tensor conformal gravity where the gravitational Lagrangian is given by

$L_{g}=\frac{1}{\alpha}\left(-\frac{1}{2} C_{\kappa \lambda \mu \nu} C^{\kappa \lambda \mu \nu}-\frac{1}{2} \nabla_{\lambda} S \nabla^{\lambda} S-\frac{1}{12} R S^{2}-\frac{\nu}{4} S^{4}\right)$,

with $S$ being the conformally coupled scalar field satisfying the scalar field equation

$\nabla_{\mu} \nabla^{\mu} S-\nu S^{3}-\frac{R S}{6}=0$,

and $\nu$ is the self-coupling parameter. In this case the field equations are given by

$W_{\mu \nu}=\frac{1}{2} S_{\mu \nu}$,

where

$S_{\mu \nu}=\nabla_{\mu} S \nabla_{\nu} S-\frac{1}{2} g_{\mu \nu} \nabla_{\lambda} S \nabla^{\lambda} S-\frac{\nu}{4} S^{4} g_{\mu \nu}+\frac{1}{6}\left(g_{\mu \nu} \nabla^{\lambda} \nabla_{\lambda} S^{2}-\nabla_{\mu} \nabla_{\nu} S^{2}+G_{\mu \nu} S\right.$

$G_{\mu \nu}$ being the Einstein tensor. The spherically symmetric exact solution to (10) and (11) obtained by Brihaye \& Verbin (2009) is given by

$d s^{2}=-B(r) d t^{2}+\frac{d r^{2}}{B(r)}+r^{2}\left(d \theta^{2}+\sin ^{2} \theta d \phi^{2}\right)$,

where

$B(r)=(1+r / a)^{2}-\frac{r_{h}}{r} \frac{(1+r / a)^{3}}{\left(1+r_{h} / a\right)}+\frac{\nu S_{0}^{2} r_{h}^{2}}{2}\left(\frac{r^{2}}{r_{h}^{2}}-\frac{r_{h}}{r} \frac{(1+r / a)^{3}}{\left(1+r_{h} / a\right)^{3}}\right)$,

and

$S(r)=\frac{S_{0}}{1+r / a}$,

where $r_{h}, a$ and $S_{0}$ are free parameters. This solution is not a generalization of the MK-solution but it is equivalent to it. In fact one can easily check that the energy momentum tensor $S_{\mu \nu}$ on the RHS of (11) vanishes for the above scalar field and metric. One can also relate the free parameters in (14) with those in the MK-solution by

$\beta=\frac{2 a r_{h}\left(a+r_{h}\right)^{2}+a^{3} r_{h}^{3} S_{0}^{2} \nu}{2\left(2 a-r_{h}\right)\left(a+r_{h}\right)^{2}-3 a^{2} r_{h}^{3} S_{0}^{2} \nu}$,

$\gamma=-\frac{1}{a}+\frac{3}{a+r_{h}}-\frac{3 a r_{h}^{3} S_{0}^{2} \nu}{2\left(a+r_{h}\right)^{3}}$

and

$k=-\frac{1}{a\left(a+r_{h}\right)}-\frac{a\left(a^{2}+3 a r_{h}+3 r_{h}^{2}\right) S_{0}^{2} \nu}{2\left(a+r_{h}\right)^{3}}$.

In his paper, Horne (2016) examined the effect of the conformally coupled scalar field $S(r)$ in (15) on the rotational curves of galaxies. In particular he showed that when the MK-metric written in the form (14) is stretched to what he calls the "Higgs frame", which is given by

$g_{\mu \nu} \rightarrow \tilde{g}_{\mu \nu}=\Omega^{2} g_{\mu \nu}=\left(\frac{S}{S_{0}}\right)^{2} g_{\mu, \nu}$,

the corresponding metric $\tilde{g}_{\mu \nu}$ lacks the linear term $\gamma \tilde{r}$ in the potential $\tilde{g}_{00}$, and therefore this has a significant effect on the shape of the resulting rotation curve in the Higgs frame geometry. Now the fact that the linear term 
in the MK-metric can be eliminated by conformal transformation has been known for a long time (see for example Mannheim \& Kazanas (1991); Schmidt (2000)) and the presence of a conformally coupled scalar field doesn't play any role in this. In fact as already remarked above and in Brihave \& Verbin (2009) the scalar field in (15) is trivial in the sense that it has no effect on the geometry because the corresponding energy momentum tensor $S_{\mu \nu}$ on the RHS of the field equations (11) vanishes. So for example starting with the MK-metric in (7) and redefining the radial coordinate by

$r=\frac{\tilde{r}}{1+\tilde{\alpha} \tilde{r}}$

the conformally transformed metric $\tilde{g}_{\mu \nu}=(1+\tilde{\alpha} \tilde{r})^{2} g_{\mu \nu}$, with $\tilde{\alpha}=\gamma /(3 \beta \gamma-2)$ takes the Schwarzschild de-Sitter form

$d s^{2}=-\tilde{B}(\tilde{r}) d t^{2}+\frac{d \tilde{r}}{\tilde{B}(\tilde{r})}+\tilde{r}^{2}\left(d \theta^{2}+\sin ^{2} \theta d \phi^{2}\right)$,

with

$\tilde{B}(\tilde{r})=1-\frac{(2-3 \beta \gamma) \beta}{\tilde{r}}-\tilde{r}^{2} \frac{\gamma^{2}-\beta \gamma^{3}+k(2-3 \beta \gamma)^{2}}{(2-3 \beta \gamma)^{2}}$

This expression now reflects the metric in Horne's Higgsfield frame, i.e. a metric conformally equivalent to that of MK by the $r$ re-scaling given in (20).

\section{Gravitational Lensing}

In further discussion in his paper, Horne (2016) argues in favor of the metric in the Higgs conformal gauge vis-á-vis gravitational lensing. It has been noted that the $\gamma r$ term in the MK metric produces a negative angle light bending, i.e. away from the gravitating source and proportional to the photon impact parameter, in gross disagreement with observation. He therefore argues that elimination of this term will reduce gravitational lensing to the standard treatment without the unwanted behavior. One has to keep in mind that unlike the case of the galactic rotational curves the effect of the linear term on the bending of light in conformal gravity is negligible and, as expected, decreases with the impact parameter. This is not surprising given that the metric obtained setting $\beta=0$ in the MK solution is conformally flat and therefore it should leave invariant the null geodesics. In fact, as shown in Sultana \& Kazanas (2010), the contribution to the bending angle from the linear term in the MK-metric is $-2 \beta^{2} \gamma / b$, where $b$ is the impact parameter, and so the ratio of this angle and the conventional bending angle $4 \beta / b$ arising from the $1 / r$ term in the metric, is of the order of $\beta \gamma$, which for a galaxy is just $\beta \gamma \sim 10^{-12}$, i.e. insignificant for practical purposes. This contrasts with earlier claims (Edery \& Paranjape 1998; Pireaux 2004a, b; Amore, Arceo \& Fernandez 2006) that this contribution from the linear term is $-\gamma b$, which becomes significant for larger values of the impact parameter $b$. These claims were based on calculations done with the improper use of the bending angle formula in spaces that are not asymptotically flat. Also apart from the negligible magnitude of the term containing $\gamma$ in the expression for the bending angle, the issue of its (negative) sign should not be considered undesirable, given that the true nature of $\gamma$ in the metric is still unknown. In fact for large $r$ the
MK-metric in (7) reduces (Mannheim \& Kazanas 1989) to a metric which is conformal to the Robertson-Walker metric with an arbitrary scale factor and with a spatial curvature that depends on $k$ and $\gamma$, which means that this parameter knows about the background spacetime and is not strictly system dependent. In other words by exploiting the conformal structure of the theory, the linear term in the metric facilitates the interpolation of the solution between the Schwarzschild and Robertson-Walker geometries in a continuous and smooth manner. The cosmological constant $\Lambda$ (which is equivalent to $k$ ) in the Schwarzschild de-Sitter geometry produces (Rindler \& Ishak 2007) a negative contribution to the bending angle. But even if one questions the validity of the cosmological nature of the parameter $\gamma$, one can still make the theory attractive to null geodesics through an appropriate choice of the conformal gauge (Edery, Mèthot \& Paraniape 2001) without the need to take $\gamma<0$ as stated in Horne (2016), and so one can still do gravitational lensing in the MK-frame. Hence one can start with the metric in (7) and apply the conformal rescaling $d s^{2} \rightarrow \tilde{\Omega} d s^{2}$ followed by the coordinate transformation

$r^{\prime}=r \sqrt{\tilde{\Omega}}$,

and assume that the conformally transformed metric has the form

$d s^{2}=-B^{\prime}\left(r^{\prime}\right) d t^{2}+\frac{d r^{\prime 2}}{B^{\prime}\left(r^{\prime}\right)}+r^{\prime 2}\left(d \theta^{2}+\sin ^{2} \theta d \phi^{2}\right)$,

where

$B^{\prime}\left(r^{\prime}\right)=1-\frac{\beta(2-3 \beta \gamma)}{r^{\prime}}-3 \beta \gamma-\gamma r^{\prime}-k r^{\prime 2}$,

contains a negative linear term. Then in a weak field approximation $\beta / r<<1, \beta \gamma<<1$, and keeping only first order terms, the necessary conformal factor is given by $\tilde{\Omega}=1-2 \gamma r$. The negative linear term in (25) will then yield a contribution to the bending angle in the direction of the object, when $\gamma>0$.

\section{Conclusion}

In this paper we have shown that one can do a simple gauge choice to transform the MK-metric in a form that does not contain the linear term and in this sense the use of a conformally coupled Higgs field to the theory and the associated Higgs frame is not required. The field itself is trivial since it yields a vanishing energy momentum tensor on the RHS of the field equations. We also point out that the sign of the linear term in the MK-metric can be changed by a similar gauge transformation and since null geodesics are preserved under conformal transformations, one can use such a gauge to obtain a positive contribution for the deflection angle, without actually eliminating it.

\section{Acknowledgments}

J.S. gratefully acknowledges financial support from the University of Malta during his visit at NASA-GSFC and the hospitality of the Astrophysics Science Division of GSFC. 


\section{REFERENCES}

Amore P., Arceo S., Fernandez F. M., 2006, Phys. Rev. D, 74, 083004

Bekenstein J. D. 2004, Phys. Rev. D, 70, 083509

Brihaye Y., Verbin Y., 2009, Phys. Rev. D, 80, 124048

Edery A., Méthot A. A., Paranjape M. B., 2001, Gen. Rel. Gravit., 33, 2075

Edery A., Paranjape M. B., 1998, Phys. Rev. D, 58, 024011

Horne K., 2016, MNRAS, 458, 4122

Kazanas D., 2009, in Contopoulos, G., Patsis, P.A., eds, Astro. Space Sci. Proceedings, Springer, Berlin, p. 485

Mannheim P. D. 1990, Gen. Rel. Gravit., 22, 289

Mannheim P. D. 1992, ApJ, 391, 429

Mannheim P. D. 1993, ApJ, 419, 150

Mannheim P. D. 1997, ApJ, 479, 659

Mannheim P. D., Kazanas D., 1989, ApJ, 342, 635

Mannheim P. D., Kazanas D., 1991, Phys. Rev. D, 44, 417

Mannheim P. D., Kazanas D., 1994, Gen. Rel. Gravit., 26, 337

Mannheim P. D., O'Brien J. G. 2011, PRL, 106, 121101

Mannheim P. D., O'Brien J. G., 2012, Phys. Rev. D, 85, 124020

McGaugh, S. 2016, PRL, 117, 201101

Pireaux S., 2004, CQG, 21, 1897

Pireaux S., 2004, CQG, 21, 4317

Rindler W., Ishak M., 2007, Phys. Rev. D, 76, 043006

Said J. L., Sultana J., Zarb Adami K., 2012a, Phys. Rev. D, 85, 104054

Said J. L., Sultana J., Zarb Adami K., 2012b, Phys. Rev. D, 86, 104009

Scarpa R., Marconi G., Gilmozzi R., 2003, A\&A, 405, L15 Schmidt H.-J., 2000, Ann. Phys. (Leipz.), 9, SI158

Sultana J., Kazanas D., 2010, Phys. Rev. D, 81, 127502

Sultana J., Kazanas D., Said, J. L., 2012, Phys. Rev. D, 86,084008

This paper has been typeset from a $\mathrm{TEX}_{\mathrm{E}}$ / $\mathrm{LT}_{\mathrm{E}} \mathrm{X}$ file prepared by the author. 